\title{
FLOW 800 for Vascular Surgery
}

\author{
Yoko Kato, Ittichai Sakarunchai, \\ and Mohsen Nouri
}

\subsection{Introduction}

Neurovascular surgeries are sophisticated procedures, and a thorough knowledge of the vessels before and during the operation is a necessity to prevent inadvertent damage and catastrophic results. Comprehensive monitoring especially real-time evaluation of cerebral blood flow is very helpful for surgery of cerebral aneurysms, cerebral arteriovenous malformations (AVMs), and extracranial-intracranial (EC-IC) bypass.

It has been shown that addition of intraoperative angiography improves the outcome of aneurysm and AVM surgeries by approving complete obliteration of the pathology and preventing inadvertent occlusion of the adjacent vessels. Until a decade ago, there was only intraoperative conventional angiography for this purpose, but recently near-infrared fluorescence module that integrated to microscope for neurosurgery was

\section{Y. Kato $(\bowtie)$}

Department of Neurosurgery, Fujita Health University, Toyoake, Aichi, Japan

e-mail: kyoko@fujita-hu.ac.jp

\section{Sakarunchai}

Division of Neurosurgery, Department of Surgery, Faculty of Medicine, Prince of Songkhla University, Songkhla, Thailand

\section{Nouri}

Gundishapour Academy of Neuroscience, Ahvaz, Iran e-mail: nouri@gan-ac.ir introduced to picture blood vessels during surgical exposure. Most commonly used fluorescent dye is indocyanine green (ICG) which was first used for assessment of hepatic function in severe chronic liver diseases for which it was approved by FDA in 1959 and later for retinal and choroidal circulation, liver and renal blood flow, and cardiac output. Microscopic-integrated nearinfrared ICG videoangiography (VA) has been used in several cerebrovascular surgeries since 2003. Intraoperative ICG-VA is done by using the commercially available microscopes (e.g., OPMI Pentero, Carl Zeiss, Oberkochen, Germany). This allows the surgeon to obtain an intraoperative angiography in less than $5 \mathrm{~min}$ (in contrast with conventional DSA which usually takes about $20 \mathrm{~min}$ ) without the need to introduce any new device (i.e., angiography unit) into the surgical field. The main shortcoming of ICG-VA is that its images are limited to the surgical field and identifying arteries from veins is not readily possible. Also, it provides the surgeon with only anatomical data without any information about the physiology and dynamics of the blood flow. To overcome these limitations, a new image analysis software package was released later to distinguish physical properties of the flow in the vessels and demonstrate semiquantitative data. 


\subsection{FLOW 800 Software}

New image analysis software, namely, FLOW 800 (Carl Zeiss, Oberkochen, Germany) was presented in 2010 that produces intensity diagrams and color mapping. This software works on the assumption that the earlier-arriving ICG in the field belongs to the arteries and those disappearing at last belong to the veins and so depicts the vascular field in a color-coded mode [1]. The operator (e.g., the surgeon or assistants) can determine some region of interest (ROI) for the software which can be further analyzed to draw the intensity diagram. This diagram shows the intensity of fluorescence over time in the ROI. More information can be deduced from this diagram after manual analysis by an image analysis software. The combination of these two processed images in accumulation to the ICG-VA helps the surgeon to judge the anatomy and physiology of the vessels in different situations (see below).

\subsubsection{Color-Coded Images}

These images convert the black and white images of conventional ICG-VA into a gradient where red and blue colors represent arteries and veins, respectively (Fig. 31.1). This can be helpful especially in AVM surgeries to identify feeding arteries. Also, if the color of a distal artery changes after clipping of an aneurysm, this might imply blood flow impairment. However, one should be cautious about interpretation of these images. As mentioned earlier, the basis of this software to color a vessel in red or blue is their chronological ICG fluorescence not the real direction of blood flow. This may result in misdiagnosis in some occasions such as when one type of vessel (artery or vein) is not in the field of view (e.g., a deep feeding artery). A good knowledge of anatomy and reviewing details of the vascular pathology before the surgery are mandatory for the surgeon to compensate for these shortcomings.

\subsubsection{Intensity Diagram}

To draw an intensity diagram, we usually use circular or rectangular marks to define the vascular area of interest (ROI), and then the curve and some primary parameters are calculated and depicted by the software. Parameters calculated directly by the software include average intensity (shown in arbitrary intensity [AI] units), delay time (i.e., the time interval from 0 to $50 \%$ of maximum fluorescence intensities [MFI]), and the slope of the curve. On the other hand, there are some other indices presented in the literature known to be correlated with perfusion characteristics of tissues which simply can be calculated with an image analysis software such as Image $\mathbf{J}$ (version 1.46, National Institute of Health, USA) after delivering the data from the microscope station (Fig. 31.2). Transit time is the time difference between MFI in artery and brain tissue, and rise time is defined as time during which fluorescence intensity rises from 10 to $90 \%$ of its peak. Other variables that can be measured manually include MFI, time to peak (i.e., from the appearance of fluorescence to MFI), and cerebral blood flow index (CBFI) which is defined as ratio of MFI to rise time. These parameters can be calculated for each vessel, and their changes should be tracked throughout the procedure [2].

\subsection{Special Application of FLOW 800}

\subsubsection{Cerebral AVMs Surgeries}

During AVM surgeries, ICG-VA can detect vessels as small as $0.5 \mathrm{~mm}$ in diameter and is very practical for in superficial AVMs. However, the field of angiography is limited to the field of microscopic view. As the surgeon cannot see the deep vessels and the whole structure of the AVM, sometimes it gets difficult to identify arteries and veins. FLOW 800 software can help the surgeon with identification of AVM vessels by producing a color-coded map where red represents feeding arteries and blue stands for drainers (Fig. 31.1). Also, after selecting some ROIs for the software, the intensity analysis curves help to understand the hemodynamics of the vessels and the surrounding brain parenchyma. For example, both image types can be used before and after temporary clipping of the arterial feeders to evaluate its effect on the AVM nidal blood flow and as a guide 

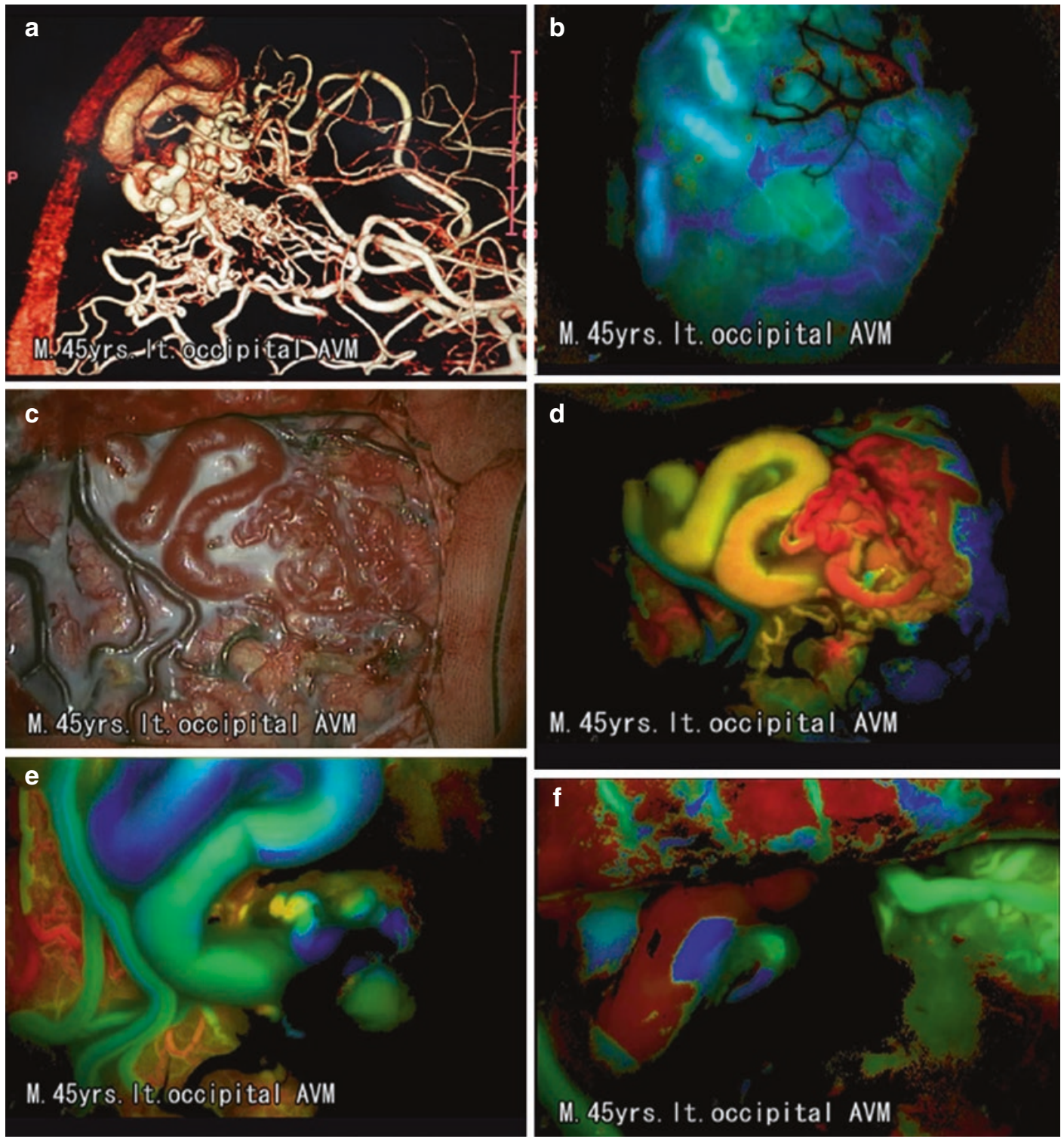

Fig. 31.1 Left occipital arteriovenous malformation (AVM) in a 45-year-old man. (a) Reconstructed computed tomography angiogram shows the location, feeding artery, and draining vein of the AVM. (b) Before opening the dura, color-coded imaging by FLOW 800 delineated vessels and helped us tailor durotomy. (c) The superficial presentation of the AVM after opening the

dura. (d) Color-coded angiography immediately after opening the dura. (e) Color-coded imaging after partial occlusion of the feeders. Note that color changes in the vessels may indicate hemodynamic changes of the AVM. (f) Color-coded angiography after confirming total resection of the malformation

for the next step of the operation. Also, changes in the cerebral parenchymal blood flow can be calculated to evaluate the effect of AVM removal on cerebral perfusion and predict postoperative autoregulation disturbances (e.g., breakthrough phenomenon). However, more data are required

to externally validate these data to better define their normal range and predictive capacity.

The main limitations of ICG-VA in AVM surgeries also extend to the FLOW 800 as deeply located vessels are not visualized and complementary DSA is required in such cases [3]. Also, 
Fig. 31.2 Intensity diagram of a vessel after manual image analysis with Image $\mathbf{J}$ software. Maximum fluorescence intensity (MFI) is the highest intensity of fluorescence for a defined area, and time to peak is the time interval from the appearance of fluorescence until its peak. Rise time is the time interval when fluorescence intensity rises from 10 to $90 \%$ of MFI. Cerebral blood flow index $(\mathrm{CBFI})$ is the ratio of MFI to rise time

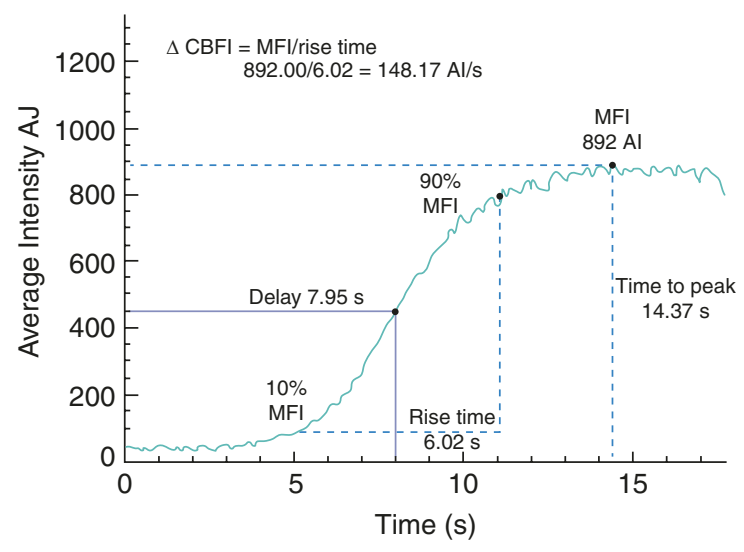

In a recent study, 2 of 12 cases needed adjustin cases in which the main vessels are covered with blood clot, brain tissue, cottonoid patties, etc., the software may not be accurate in differentiating arteries from veins.

\subsubsection{Cerebral Aneurysm Surgeries}

In aneurysm surgeries, ICG-VA can demonstrate perforating arteries before and after clipping, check for patency of the distal vessels after clipping, and confirm complete aneurysm obliteration. However, there are certain circumstances where checking the anatomy with ICG-VA is not all enough for a safe surgery. Sometimes, despite having the distal blood flow depicted on angiograms, subtle decrease in flow presents which may compromise perfusion of the cerebral parenchyma leading to disturbed function of the brain. FLOW 800 may assist the surgeon to calculate the CBFI before and after clipping and by showing him or her the color-coded images where any change in color after clipping regarded as an alarming sign.

When ICG-VA is repeated at surgery after clipping, the dome of the aneurysm may show some residual ICG illumination from previous injections. This results in confusion as the surgeon cannot be assured of the total obliteration of the neck. In this occasion, FLOW 800 can differentiate a residual ICG from incomplete clipping: in case of clip shortage, the aneurysm dome appears red in color-coded images, and if the dome is selected as a ROI, a curve that follows an arterial diagram is produced (Fig. 31.3). ment of the clip after finding an occlusion of perforating vessels [4]. Also, after ICG-VA they found 4 cases of incomplete clipping out of 45 $(8.9 \%)$ who required appropriate readjustment to complete the obliteration.

In some cases, atherosclerotic plaque in the aneurysm may make interpretation of ICG-VA very difficult if not impossible. In these cases when there is any doubt in the result of an ICG-VA, we should use other monitoring techniques such as micro-doppler or endoscope that can be used to check for residual blood flow in aneurysm sac or incomplete obliteration of the aneurysm neck.

One of the advantages of ICG-VA over DSA in aneurysm surgery is its real-time nature where the surgeon can manipulate the vessels and adjust the clip just during the angiography, if required. Also, FLOW 800 allows physiological monitoring of the clipping procedure both in the vessels and in the surrounding brain tissue. ICG-VA has largely replaced conventional DSA during aneurysm surgery in most centers, and attempts to further clarify the role of FLOW 800 in these procedures are underway.

\subsubsection{Bypass Surgeries}

Different stages of EC-IC bypass surgery such as to identify the recipient artery, to evaluate patency of the graft, and to detect any possible stenosis are facilitated by ICG-VA. Januszewski 

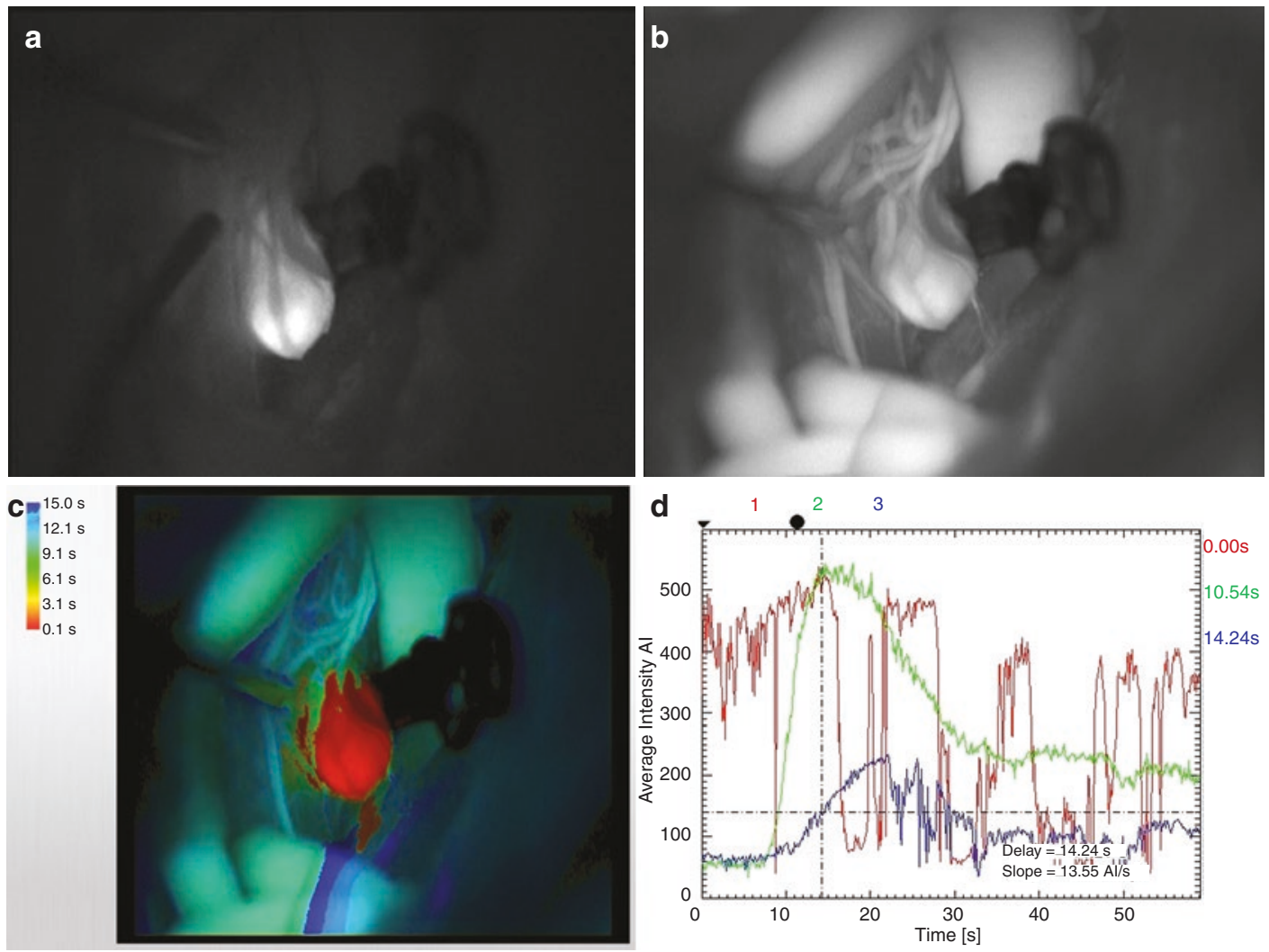

Fig. 31.3 (a) Surgical view under infrared camera after aneurysm clipping and before indocyanine green (ICG) injection. Note that the aneurysm glows due to previous ICG accumulated in the aneurysm sac. (b) The same view after ICG injection. It is difficult if not impossible to differentiate previously accumulated ICG in the aneurysm from newly injected in the vessels. This view is consistent with incomplete aneurysm obliteration. (c) Color-coded image by FLOW 800 software demonstrate the aneurysm

in red. This is the shortcoming of the software that cannot differentiate a previously injected dye from early arrival of blood flow. (d) The red curve shows the intensity changes inside the aneurysm sac. Constant curve confirms absence of blood flow in the aneurysm. Some sudden changes in intensity are observed which are due to manipulation of the aneurysm with suction tip to see behind the aneurysm. Please note that delay and slope of the curve are calculated by the software

et al. used ICG-VA to analyze the blood flow type after anastomosis which can predict early postoperative graft occlusion [5]. Also, Esposto et al. used ICG-VA to identify arterial territory in temporary or permanent occlusion of the vessels [6]. Although the application of the ICG-VA in bypass evaluation is well established and widely accepted, the role of FLOW 800 is still unclear. In spite of some available limited data [7], whether pre- to post-bypass, ratio of measures such as CBFI or MFI correlates with the outcome and requires further comparative studies.

\subsection{Expert Opinion}

Additional hemodynamic analysis with the help of FLOW 800 to the conventional angiographies (e.g., ICG-VA) allows a real-time physiological and anatomical assessment of blood flow during cerebrovascular procedures such as aneurysms, AVMs, and bypass surgeries. Intraoperative imaging of the vessels in vascular surgeries decreases postoperative morbidities and is an inseparable adjunct in almost all centers dealing with such pathologies. This along with other 
intraoperative monitoring modalities such as neuroendoscopy and motor-evoked potentials results in superb outcome of the patients in the modern era of cerebrovascular surgeries [8]. Intraoperative DSA is still considered the gold standard procedure especially for AVMs where the deep vessels pose certain concerns during the surgery. However, in most aneurysm and selected AVM surgeries, ICG-VA can replace conventional DSA as it is a cheaper technique, requires less time for the procedure, and eliminates exposure to radiation. Also, physiological evaluations by FLOW 800 are very useful for objective documentation of the blood flow in aneurysm sac, AVM vessels, and bypass graft. Yet, as these data are semiquantitative, external validation of these measures against standard physiological assessments is required to better clarify the value of the numbers calculated by the software and how they can be applied in practice. This will be the topic of future studies.

\section{References}

1. Oda J, Kato Y, Chen SF, Sodhiya P, Watabe T, Imizu S, Oguri D, Sano H, Hirose Y. Intraoperative near-infrared indocyanine green-videoangiography (ICG-VA) and graphic analysis of fluorescence inten- sity in cerebral aneurysm surgery. J Clin Neurosci. 2011;18:1097-100.

2. Son YJ, Kim JE, Park SB, Lee SH, Chung YS, Yang HJ. Quantitative analysis of intraoperative indocyanine green video angiography in aneurysm surgery. J Cerebrovasc Endovasc Neurosurg. 2013;15(2):76-84.

3. Ye X, Liu XJ, Ma L, Liu LT, Wang WL, Wang S, Cao Y, Zhang D, Wang R, Zhao JZ, Zhao YL. Clinical value of intraoperative indocyanine green fluorescence video angiography with Flow 800 software in cerebrovascular surgery. Chin Med J. 2013;126(22):4232-7.

4. Chen SF, Kato Y, Oda J, Kumar A, Watabe T, Imizu S, Oguri D, Sano H, Hirose Y. The application of intraoperative near-infrared indocyanine green videoangiography and analysis of fluorescence intensity in cerebrovascular surgery. Surg Neurol Int. 2011;2:42.

5. Januszewski J, Beecher JS, Chalif DJ, Dehdashti AR. Flow-based evaluation of cerebral revascularization using near-infrared indocyanine green videoangiography. Neurosurg Focus. 2014;36(2):1-11.

6. Esposito G, Durand A, Doormaal TV, Regli L. Selective-targeted extra-intracranial bypass surgery in complex middle cerebral artery aneurysms: correctly identifying the recipientartery using indocyanine green videoangiography. Neurosurgery. 2012;71(ONS Suppl 2):274-85.

7. Uchino H, Nakamura T, Houkin K, Murata J, Saito H, Kuroda S. Semiquantitative analysis of indocyanine green videoangiography for cortical perfusion assessment in superficial temporal artery to middle cerebral artery anastomosis. Acta Neurochir. 2013;155(4):599-605.

8. Yamada Y, Kato Y, Nouri M. No overtaking! Take a safe trip to aneurysm. Austin J Cerebrovasc Dis Stroke. 2014;1(2):1.

Open Access This chapter is licensed under the terms of the Creative Commons Attribution 4.0 International License (http://creativecommons.org/licenses/by/4.0/), which permits use, sharing, adaptation, distribution and reproduction in any medium or format, as long as you give appropriate credit to the original author(s) and the source, provide a link to the Creative Commons license and indicate if changes were made.

The images or other third party material in this chapter are included in the chapter's Creative Commons license, unless indicated otherwise in a credit line to the material. If material is not included in the chapter's Creative Commons license and your intended use is not permitted by statutory regulation or exceeds the permitted use, you will need to obtain permission directly from the copyright holder. 